\title{
A community-centric approach to automated service composition
}

\author{
LIU XuanZhe
}

In recent years, the Internet has been evolving from a primarily publication platform to a user participatory platform. With the proliferation of services available on the Internet, millions of users are able to voluntarily participate in the development of their own interests and benefits by means of service composition. However, due to the ever increasing number of services, enabling users to rapidly select and access these services has become a challenging issue.

It should be noted that the innovations available from Web 2.0 standard have brought fresh and user-centric experiences. For any given topic or interest (such as traveling, photography or reading), the users may organize themselves into a "community-of-interest" set, where they can share their interests, exchange ideas, and acquire enriched solutions, thus giving rise to a "collective intelligence". Typical examples are the successful social-networking web sites such as Facebook, Flickr and Del.icio.us. Such community-centric environments may also have impacts on service-based software development.

In this work, the notion of "service communities" is proposed to facilitate automated service access. In summary, service communities address the issue of accessing a large and ever changing collection of services. Logically, it provides a platform which assists users with similar interests or goals to retrieve, share and reuse existing available solutions. By interacting with a service community, users are able to build their own applications with less effort and cost, and iteratively refine their goals according to the combined available knowledge.

Apart from conceptual ideas, this work focuses on service community consolidation, with the design of the architectural framework of a service community. Two important components, in particular, are identified: a service pool aggregating a set of services offering the same or similar functions, so that users do not have to drill down into details of individual services; and a task template capturing the access logic of some specified task, which explores the potential access opportunities and assists in accessing the service.

Supporting automated service access, the service community helps the users by providing two composition styles: the process-driven manner allowing users to reuse existing access processes, and the user-driven manner enabling users to iteratively access new processes in a step-by-step manner. A graphical model is employed to generate access suggestions to users.

A web-based prototype is implemented to evaluate the community-centric service access approach. The users can visually access services within web browsers. Solutions from the service community dynamically result in changes to the user interface.

A journal reviewer stated, "This paper proposes a precise and feasible solution to service-based software development with the adoption of community-generated intelligence". In addition, it was noted, "The authors made an effort to provide conceptual proof and solid implementation". A series of papers about "community-centric service composition" written by Dr. Liu, Prof. Huang, Prof. Mei has been published in IEEE Trans Serv Comput., Sci China Inf Sci, etc. Another reviewer commented "The paper enriches research results on services computing as well as showing the potential emerging software development paradigm spurred by the Internet evolution".

The authors are affiliated to The Key Laboratory of High Confidence Software Technologies, Ministry of Education (Peking University). This laboratory is conducting research mainly in the fields of high-confidence software fundamental theory, software engineering, and middleware technologies.

See the articles: Liu X Z, Huang G, Mei H. A community-centric approach to automated service composition. Sci China Inf Sci, 53: 50-63

Liu X Z, Huang G, Mei H. Discovering homogeneous web services community in the user-centric web environment. IEEE Trans Serv Comput, 2009, 2: 167-181 